\title{
影响多层交流超导电缆电流分布不可忽略的 因素-邻近效应
}

信赢"，龚伟志，张敬因

北京云电英纳超导电缆有限公司, 北京 100176

*E-mail: yingxin@innopower.com

收稿日期: 2009-12-16; 接受日期: 2010-01-22

国家高技术研究发展计划(“863”计划)(批准号：2002AA306154，2004AA306110，2005AA306120)和北京市重大科技项目(批准号： H020420010790, H020420010210)资助项目

\begin{abstract}
摘要 零电阻是超导体的本征特性之一. 人们在努力寻求超导材料零电阻特性带来的有利效 益时, 也要研究其可能导致的一些对我们不利的影响. 在由高温超导材料制作的多层交流超导电 缆中, 由于超导体的零电阻特性而导致邻近效应异常强烈. 这种强烈的邻近效应会使电缆中的电 流分布变得严重不均匀，对电缆的性能产生负面影响。在这篇文章里，我们将报道多层交流超导 电缆中强邻近效应现象及其对电缆层电流分布的影响. 也许可以把超导体产生的这种非常强的邻 近效应称作“超强邻近效应”. 认识这一现象也会对涉及交变电流的其他超导应用提供有益的启示.
\end{abstract}

关键词 超导电缆 电流分布 邻近效应 高温超导

\section{1 前言}

一个导体内的交变电流会在其附近产生磁场, 影响其相邻导体中的电流分布, 可能导致电流集中 在导体中的部分区域、或电流路径紊乱、涡流等情况 的发生, 这种现象称作邻近效应. 邻近效应会使导体 有效的通流面积减小, 增加电流的传输损耗, 降低导 体传输电流的能力 ${ }^{[1,2]}$.

在许多使用多股导体的应用中，譬如在设计电 力电缆、变压器绕组、声频电缆时, 都要考虑邻近效 应的影响 ${ }^{[3 \sim 6]}$. 邻近效应也会影响到一些超导材料的 自身性质和超导器件的性能 ${ }^{[7 \sim 9]}$.

邻近效应本质上是多个相邻导体中交变电磁场 相互作用对电子在导体中的运动产生影响的结果. 它与输送电流的频率、导体性质、导体的尺寸及空间 位置等因素都有关系.
到目前为止，人们还没有充分认识邻近效应对 高温超导电缆的影响. 与常规电缆及低温超导电缆 使用多股线的导体结构不同 ${ }^{[10 ~ 13]}$, 高温超导电缆一 般采用分层的导体结构 ${ }^{[14 ~ 18]}$. 人们在设计多层高温 超导电缆时, 通常主要考虑如何平衡各个导体层的 电感和尽量降低载运交流电时的磁滞消耗, 而忽略 了邻近效应的影响 ${ }^{[19 \sim 24]}$.

我们在进行多层导体结构的高温超导电缆的交 流通流实验研究中, 发现邻近效应严重影响超导电 缆各层导体中的电流分布.

\section{2 多层高温超导电缆的层电流分布实验}

\section{1 实验样品的描述}

为研究具有多层导体结构交流超导电缆中的电 
流分布规律, 我们先后制作了 3 个长为 $3 \mathrm{~m}, 6$ 层导体 结构的超导电缆样品. 这些样品的导体结构如图 1 所 示. 其中, 各层导体由超导带材按不同的绕制方向、 角度绕制而成, 层间用 $50 \mu \mathrm{m}$ 聚酰亚胺薄膜绝缘. 使 用的超导带材为银包套 $(\mathrm{Bi}, \mathrm{Pb})_{2} \mathrm{Sr}_{2} \mathrm{Ca}_{2} \mathrm{Cu}_{3} \mathrm{O}_{10}$ 带材, 宽为 $3.8 \sim 4.0 \mathrm{~mm}$, 厚为 $0.18 \sim 0.20 \mathrm{~mm}$, 超导带材同轴 绕制在外径为 $30 \mathrm{~mm}$ 的支撑管(不锈钢波纹管)上. 样 品 1 和 2 的具体绕制参数及部分电气参数分别由表 1 和 2 给出.

为使超导电缆的导体使用效率最高, 通流能力 最大, 交流损耗最小, 我们希望各层导体能够均匀通 流. 按照通常的认识, 并联支路中的电流分配决定于 各支路的阻抗. 而各支路的阻抗是由其电阻、电感和 电容决定的.

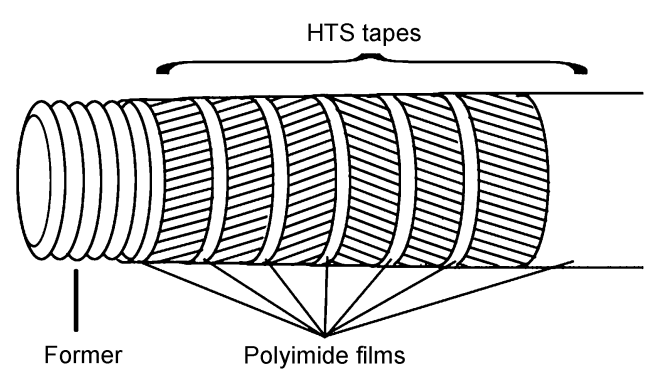

图 1 多层高温超导电缆导体结构

\section{表 1 样品 1 的制作参数及各层电阻值}

\begin{tabular}{lllllll}
\hline 导体层 \# & 1 & 2 & 3 & 4 & 5 & 6 \\
\hline 绕制角度 $\left(^{\circ}\right)$ & 37.3 & 30.0 & 18.2 & 20.1 & 33.5 & 43.9 \\
带材根数 & 15 & 17 & 20 & 21 & 19 & 17 \\
绕制方向 & + & + & + & - & - & - \\
室温电阻 $(\mathrm{m} \Omega)$ & 6.4 & 5.1 & 4.0 & 3.9 & 4.8 & 6.2 \\
\hline
\end{tabular}

“+”代表顺时针绕制, “-”代表逆时针绕制, “导体层\#”1,2, “, 6 的顺序为从最内层至最外层.

表 2 样品 2 的制作参数、各层电阻值、电感值及感抗值

\begin{tabular}{lllllll}
\hline 导体层 \# & 1 & 2 & 3 & 4 & 5 & 6 \\
\hline 绕制角度 $\left({ }^{\circ}\right)$ & 32.5 & 24.9 & 19.2 & 15.1 & 13.1 & 10.0 \\
带材根数 & 18 & 19 & 19 & 19 & 18 & 18 \\
绕制方向 & + & + & + & + & + & + \\
室温电阻 $(\mathrm{m} \Omega)$ & 5.0 & 4.4 & 4.3 & 4.2 & 4.4 & 4.3 \\
电感 $(\mu \mathrm{H})$ & 23.5 & 19.8 & 19.8 & 16.0 & 15.9 & 24.8 \\
感抗 $(\mathrm{m} \Omega, 50 \mathrm{~Hz})$ & 8.53 & 6.23 & 6.22 & 5.02 & 4.99 & 7.80 \\
\hline
\end{tabular}

电阻是导体导电能力的量度. 一个电路里电阻 的大小取决于其导体的电阻率、导体几何尺寸及其 连接方式. 对于我们的样品, 每一层导体的电阻只 与带材的线电阻率、根数及长度有关. 而带材的长度 是由绕角决定的. 绕角越大, 带材就越长. 带材越长, 电阻就越大. 因带材之间的关系是并联, 故一般来 讲, 某一层带材的根数多, 其层电阻就会相对小一 些. 电缆处于常导态时, 各层导体均有一定的电阻, 必须考虑电阻对电流分配的影响. 而处于超导态时, 样品电缆的各层导体直流电阻为零, 对电流分配没 有影响.

电感是指在交流电的作用下, 电路通过电磁感 应产生反电动势能力, 这与导电线路的自身结构相 关. 在我们的样品中, 绕角越大的导体层, 其自感也 越大. 各导体层之间的互感则不但与每层导体的绕 角，而且与它们之间的相对方向有密切关系.

电容指线路储存电量的能力, 它与电缆的体积、 形状、绝缘介质和环境参数有关. 在我们的实验样品 中, 无论在常导态还是超导态, 电容的影响都可以忽 略不计.

因此, 根据常规的电路计算, 在超导电缆处于常 导态时, 电流的分配取决于电阻和电感两个因素, 而 处于超导态时, 只取决于电感的作用. 很多人认为超 导电缆中决定电流分布的因素主要为各层的自感和 它们之间的互感, 各层只要通过合理的绕制角度设 计，使每层的自感、互感之和基本相同，那么完全可 以实现电流的均匀分布 ${ }^{[15,20,23,25]}$. 我们在样品 1 的设 计工作中也是本着这样的原则, 在确定每层带材的 缠绕角度和方向时, 力求保证每层的感抗(自感+互 感)相等(每一层电感的设计值为 $8 \mu \mathrm{H}$ 左右), 这样在 进入超导态后, 电缆能够实现层间均流. 在制作过程 中由于加工公差的存在, 不同层的电感值有微小的 差别 $(<4 \%)$.

因为样品 1 的试验结果出人意料, 在超导态并没 有达到均流的效果, 所以我们开始怀疑层电感在决 定超导态电流分配上的作用. 为了回答这个问题, 我 们设计和制作了样品 2. 样品 2 的特征是各层的缠绕 方向相同, 缠绕角度由里向外逐渐减小. 第一、五、 六层超导带材的根数均为 18 根, 而第二、三、四层 则均为 19 根. 为了验证电感的作用, 样品 2 各层的电 感设计了较大的差异. 
在样品 2 试验完成以后, 实验结果否定了电缆层 电感对超导态电流分布的决定作用，同时发现了一 些具有规律性的现象. 为了证实这些规律, 我们又设 计、制作了样品 3. 样品 3 每层的根数及缠绕角与样 品 1 相同, 即每一层的电组特性与样品 1 相同, 但缠 绕方向为+-+-+-, 即层与层交替变化的, 与样品 $1+++$, ---不同. 不难想象样品 3 的层电感与样品 1 和样品 2 的层电感都会有明显差别的. 因为在前两个样品的 实验中已排除了电感的决定性作用, 故没有对样品 3 的层电感做详细的设计和测量.

\section{2 层电流分布测量的实验仪器与方法}

测量样品层电流分布的实验电路如图 2 所示. 样 品的各层导体均通过铜编织带并联到电流引线铜排 上. 为测量各层导体分电流及线路的总电流，在铜编 织带及铜排上均装有罗高斯线圈, 并通过 HP34970 巡检采数. 实验电源为 $220 \mathrm{~V}$ 工频 $50 \mathrm{~Hz}$ 单相电源, 通 过一个 $4 \mathrm{~V} / 5000 \mathrm{~A}$ (有效值, 本文所谈及的电流值均为 交流电流的有效值)降压变压器提供电流, 实验中超导 电缆的通流可达 4000 A. 我们还使用 Kethley2000 和 锁相放大器 SR850 测量每层的电压及其对于电流之 间的相角. 实验时, 整个超导电缆样品放置在一个长 约 $4 \mathrm{~m}$ 的液氮槽内, 先给线路通电, 再缓慢注入液氮, 这样可以观测到超导电缆由常导态转变至超导态整 个过程中电气参数的变化.

\section{3 实验过程与结果}

实验是在样品从室温(约 $295 \mathrm{~K}$ ) 向一个大气压下 的液氮温度 (约 $77.3 \mathrm{~K}$ ) 过渡的过程中进行的. 实验开

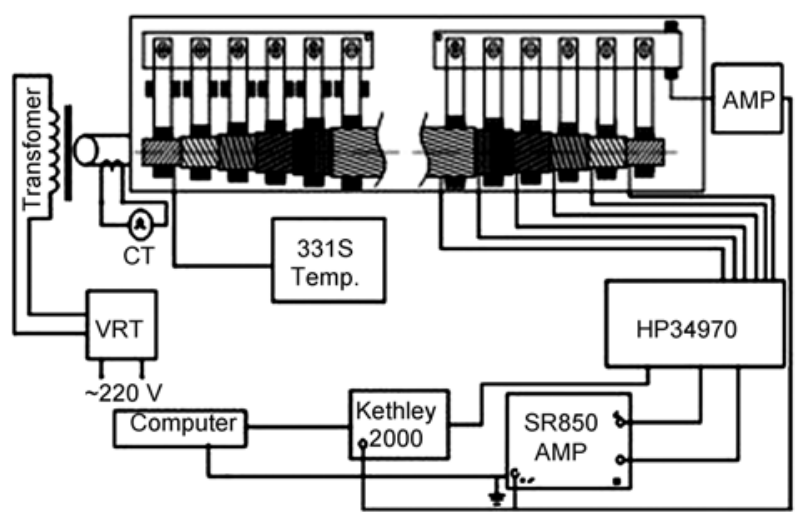

图 2 样品层电流分布测量电路
始时, 往液氮槽内注入液氮, 约两分钟后调节供流变 压器, 给样品通 $100 \mathrm{~A}$ 的测试电流, 并开始层电流分 布的测量. 随着样品温度的下降，电阻也随之下降， 测试电流不断增大. 因液氮槽、样品支架及样品的热 容量很大, 所以试验的最初阶段, 大量的液氮都汽化 蒸发了. 约数十分钟后, 液氮才把样品浸没, 此后样 品的温度保持在液氮温度. 测量第一个样品时样品 被冷却到液氮温度用了约 $35 \mathrm{~min}$, 测量后两个样品 时, 加大了液氮注入流量, 冷却样品的时间缩短到 $20 \mathrm{~min}$ 以内. 在冷却过程中，降温的速率是不均匀的, 最初比较慢, 后来逐渐加快.

图 3 显示了样品 1 在常导态及超导态时各层导体 中电流分配的状况. 首先看常导态的情况, 这时层电 流的分布相对来说是比较均匀的, 六层导体每层通 过的电流为总电流的 $15 \sim 18 \%$. 设第 $n(n=1,2, \cdots, 6)$ 层 通过的层电流为 $I_{n}$, 如果我们按层电流的大小做一个 排序, 则有 $I_{4}>I_{3}>I_{5}>I_{2}>I_{6}>I_{1}$.

对于样品 1 来说, 因各导体层的感抗大致相等, 所以其按阻抗大小的排列顺序与各导体层的按电阻 大小的排列顺序是一致的. 根据表 1 , 我们可以得出 $Z_{1}>Z_{6}>Z_{2}>Z_{5}>Z_{3}>Z_{4}\left(Z_{n}, n=1,2, \cdots, 6\right.$, 是每一导体层的 阻抗). 这个样品中每层阻抗大小的顺序与常导态层 电流分布顺序的测试结果是相吻合的.

这里还要指出, 每个导体层两端的接头电阻的 阻值若有较大的差异，也会对层电流的分布产生影 响. 从常导态层电流分布的测试结果来看, 各层的接 头电阻的阻值差异要远比每一层的电阻值小.

当随着温度降低到超导电缆的临界转变温度之 下，超导带材进入无电阻的超导态. 从简单的电工电 路角度来考虑, 这时候影响电缆层电流分布的因素 就只剩下每一导体层的感抗和接头电阻了. 前面说 过样品 1 的各层感抗的差别不大, 这样在进入超导态 后, 如果接头电阻的影响可以忽略, 那么这个电缆样 品应该能够实现层间均流。

那么, 接头电阻在这里是否能起到决定性的作 用呢? 我们对接头电阻做了测量, 接头电阻的绝对 数值很小 (为 $10^{-6} \sim 10^{-5} \Omega$ ), 且各个接头的差异也很小 $\left(<10^{-6} \Omega\right.$ 量级). 而每一层的感抗在 $10^{-3} \Omega$ 的数量级, 各层间 $1 \%$ 的感抗差异也要比接头电阻至少高出一个 数量级. 排除了接头电阻可能的影响, 所能直接想到 的影响层电流分布的主要因素就只剩下感抗了. 而 样品 1 各层的感抗大致相等, 故我们期待在超导态它 
的各层的电流大致相等, 实现均流的效果.

可是实验结果却出乎意料. 从图 3 可以看出, 在 电缆样品进入超导态后, 层电流不但没有按照设计 向更均匀的方向发展, 而是出现了非常不均匀的分 布. 其中最大的层电流达到电缆总电流的近 $25 \%$, 而 最小的层电流只有总电流的约 $9 \%$. 这时层电流按大 小的排序为 $I_{6}>I_{1}>I_{5}>I_{2}>I_{4}>I_{3}$, 和常导态时的排序 $I_{4}>$ $I_{3}>I_{5}>I_{2}>I_{6}>I_{1}$ 是大相径庭的. 第 6 层由常导态时的次 小跃升为超导态时的最大, 第一层由常导态时的最 小变为超导态时的次大. 而第四层和第三层则分别 由原来的最大和次大颓变为次小和最小. 如果单纯 地从每一层的感抗来考虑, 这样的结果是绝对不能 接受的. 因为各层感抗的差异决不会有这么大, 这在 常导态的结果中已经得到了验证.

在超导态，我们还对该样品进行了不同输运电 流条件下层电流分布的测试, 图 4 给出相关实验结果. 可以看出从电缆电流 $350 \mathrm{~A}$ 到 $3000 \mathrm{~A}$, 层电流分布顺 序没有改变.

样品 1 的实验结果出乎意料, 为了搞清楚究竟决 定超导电缆层电流分布的主要因素是什么, 我们开 展了进一步的实验工作. 我们又制作了一个超导电 缆样品, 即样品 2 , 其长度、导体层数、及导体内、 外径均与样品 1 相同, 其他设计、绕制等参数已由表 2 给出.

图 5 给出为样品 2 常导态及超导态时电流分布测 量结果. 电缆样品处于常导态时, 层电流分布规律与 常规的电路计算能够很好地符合. 最内层导体的电 阻、感抗最大、最外层次之. 实验结果显示, 常导态 时, 最内层分流最小, 为 $12 \%$. 最外层次小, 为 $13 \%$. 电阻及感抗均最小的第 4 层分流量最大, 为 $22 \%$. 样 品 2 各层的电阻、电感不同, 常导态下, 层电流分布 差别明显，范围增至 12 22\%.

与样品 1 类似, 在进入超导态后, 样品 2 的层电 流大小和相互排列顺序发生了剧烈的变化, 按大小 排序为 $I_{6}>I_{5}>I_{1}>I_{2}>I_{3}>I_{4}$. 很明显, 感抗并没有对层电 流分布产生决定性的影响, 这与样品 1 的实验结果是 吻合的. 另一个与样品 1 超导态类似的结果是层电流 大小的分布顺序.

为了得到更多的实验数据, 验证在前两个样品 实验中似乎得到的规律性现象, 我们又设计、制作了 第三个样品. 图 6 是样品 3 的电流分布测量结果, 在
常导态时的层电流分布顺序为 $I_{1}>I_{2}>I_{4}>I_{3}>I_{6}>I_{5}$. 因 样品 3 的层电阻应与样品 1 的层电阻基本相同, 而常 导态的层电流分布却与样品 1 不同, 这就说明其层感 抗差异在层电流分配上起了明显的作用. 样品 3 常导 态的层电流分布范围为 14 20\%，大于样品 1 的 15 $18 \%$ 的范围, 但小于样品 2 的 12 22\%的范围.

在进入超导态后, 样品 3 的层电流分布同样也发 生了急剧的变化, 其大小顺序为 $I_{6}>I_{1}>I_{5}>I_{3}>I_{2}>I_{4}$.

在样品 2 和样品 3 的层电流实验中, 可以清楚地 看出绕制参数的变化给常导态层电流分布带来的显 而易见的变化，这种变化对应着层电阻和层电感的 变化.

那么在超导态我们可以得到什么结论呢? 为此 我们把三个样品层电流分布的结果放到一起，从中 找到一些规律性的东西. 图 7 给出了三个样品超导态 时层电流大小的排序情况. 尽管三个样品的缠绕方 式截然不同, 缠绕角度相差很大, 常导态的层电流分 布大不相同，但电缆进入超导态后却出现了一个相 同的趋势, 无一例外地出现了最外层和最内层电流 比较大, 中间层电流比较小的现象. 对于三个样品来 说都是最外层, 即第 6 层的层电流最大, 其中样品 1 和样品 3 的最里层(第 1 层)为次大, 样品 2 的最里层 为第三大. 但如果再仔细观察反映样品 2 的层电流分 布情况的图 5, 我们会发现其第 1 层的层电流与位居 次大的第五层层电流的差别是很小的. 再看层电流 最小的情况, 样品 1 和样品 2 层电流最小的两层是夹 在中间的两层, 即第三层和第四层, 样品 3 第二层的 层电流虽然比第三层小, 但从图 6 可以看出两者相差 不大. 所以基本可以说尽管三个样品的层感抗分布 有很大差异, 但在超导态的层电流分布方面却有着 很强的相似性. 这种相似性可以归纳为：一般地说, 最外层的层电流最大, 最内层的层电流次之; 夹在中 间的两层层电流最小; 次外层和次内层层电流居中, 但次外层的层电流相比要大一些.

我们还注意到在超导态时三个样品的层电流比 例分布范围都很大，分别约为 9 25\%，10 28\%，和 $8 \sim 28 \%$. 也就是说最大值是最小值的 3 倍左右. 而样 品 1 的层电感基本是相同的, 样品 2 或样品 3 的虽然 不同，但其大小和相对应层电流的大小的关系是违 背电路的一般规律的. 所以产生这样的层电流分布 情况的主要因素决不是每层的感抗. 


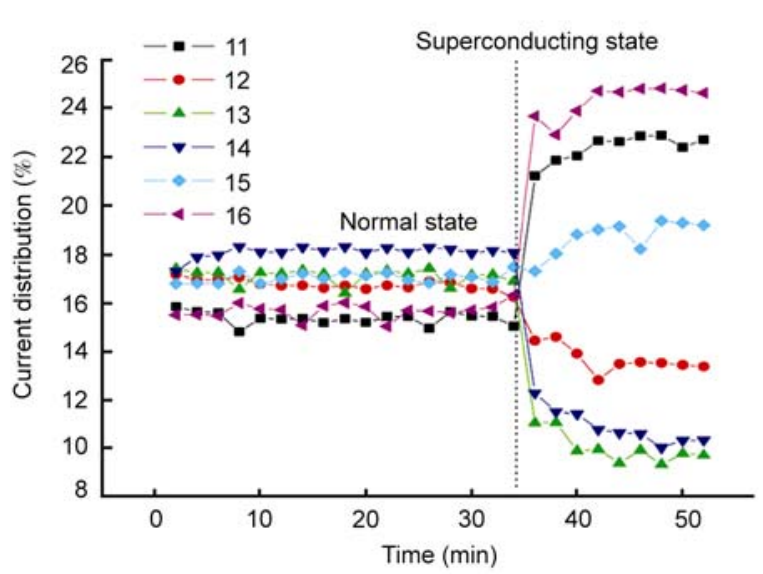

图 3 样品 1 层电流分布测量结果

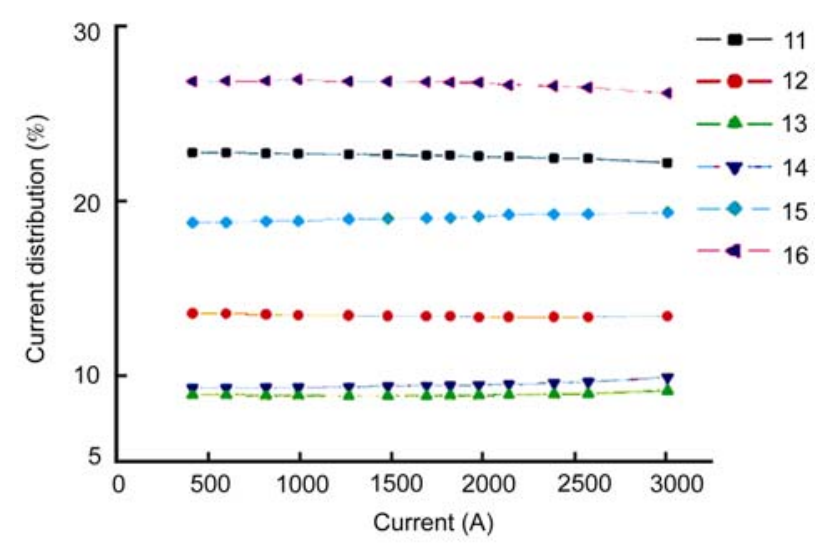

图 4 在不同输运电流条件下样品 1 层电流分布

\section{4 超导导体的邻近效应}

究竟是什么因素决定了三个样品的层电流分布 特性呢? 在排除了一些能够想到的可能性之后, 我 们把注意力集中在了邻近效应上面.

邻近效应的起源是由于导体中交变电流激励的 磁场对电子在导体的运动产生影响, 使导体电流密 度的分布变得不均匀. 为分析超导电缆中的邻近效 应, 我们首先分析一个简单的例子：一对相距很近的 平行金属板通过交流电流时的情况. 图 8(a)中，一对 宽度为 $a$, 高度为 $b$ 的长金属板相距为 $d$, 如图平行放 置，且都通过交流电流 $I$. 交流电流会在金属内及其 附近的空间感应出交变磁场, 在两金属板相距较远 $(d$ 较大)时, 一个金属板上的电流产生的磁场不会波及 到另一个金属板, 所以两个金属板内的电流都是相

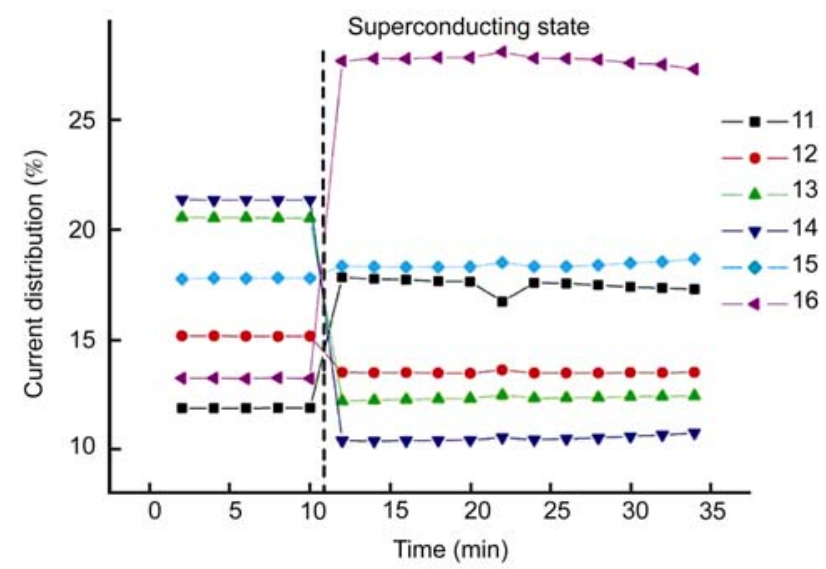

图 5 样品 2 层电流分布测量结果

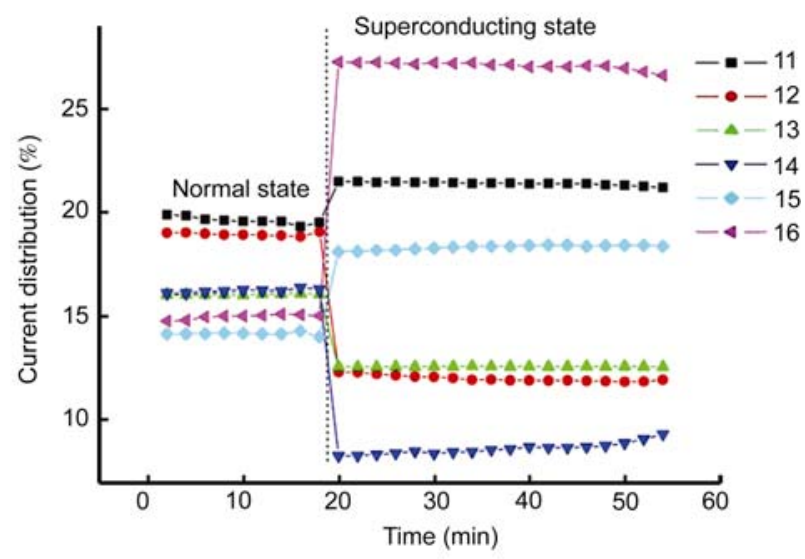

图 6 样品 3 层电流分布测量结果

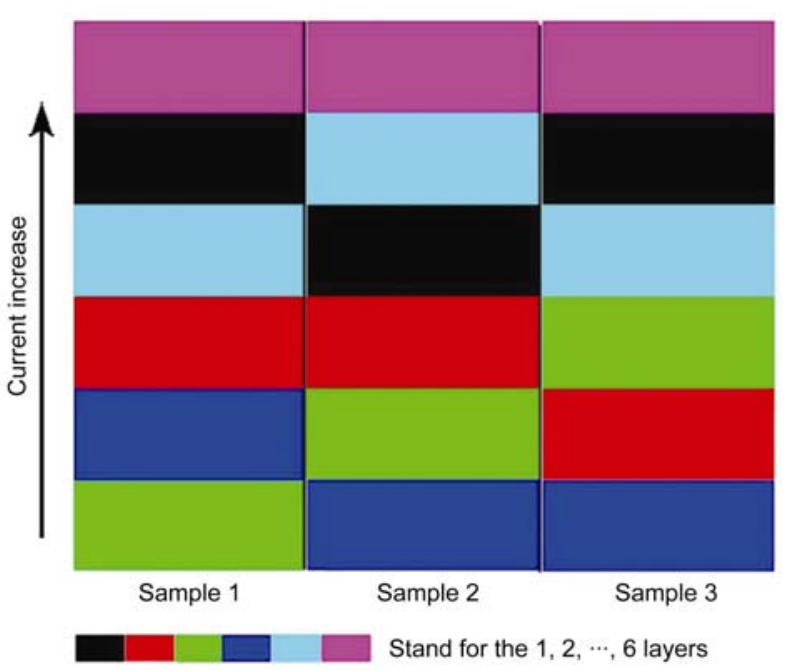

图 7 三个样品超导态层电流分布大小顺序排列 
对各自横截面的中线对称分布的. 但当 $d$ 很小时, 一 个金属板里的磁场的大小和方向不但取决于其上流 过的电流, 而且还受另一金属板上电流的影响. 这种 磁场和电流的作用满足 Maxwell 方程组:

$$
\left\{\begin{array}{l}
\nabla \times \dot{\boldsymbol{H}}=\dot{\boldsymbol{J}}+\mathrm{j} \omega \dot{\boldsymbol{D}}, \\
\nabla \times \dot{\boldsymbol{E}}=-\mathrm{j} \omega \dot{\boldsymbol{B}} .
\end{array}\right.
$$

从(1)和(2), 我们得到

$$
\left(\mathrm{j} \omega \sigma \mu_{0}-\omega^{2} \mu_{0} \varepsilon_{0}\right) \dot{\boldsymbol{H}}-\nabla^{2} \dot{\boldsymbol{H}}=\mathbf{0} .
$$

根据已知条件和边界条件, 方程(3)的解为

$$
J_{x}=\frac{I}{b \sinh (k a)} k \cdot \cos h k\left(\frac{d}{2}-x\right) .
$$

这里 $J_{x}$ 是金属板内位于 $x$ 处的电流密度, $k^{2}=\mathrm{j} \omega \sigma \mu_{0}, \mathrm{j}$ 是虚数单位, $\omega$ 是交流电流的角频率, $\sigma$ 是金属板的电 导率, $\mu_{o}$ 是真空磁导率.

因为金属板里的自由电子在这个交变磁场中会 受到一个与电流方向垂直 ( $x$-轴方向)的力 (洛仑兹力) 的作用, (4)式所表示的电流密度 $J_{x}$ 已经不再是常数. 为了展示金属板的电导率是如何影响电流分布的, 图 8(b)给出了对应四种不同电导率, 两个金属板中电 流密度的分布情况. 我们假设当电导率为 $\sigma$ 时 $J_{x}$ 曲线 可以用图中红色的曲线表示. 如果电导率变化为 $2 \sigma$, $J_{x}$ 分布就会遵循棕色曲线. 绿色曲线代表电导率是 $4 \sigma$ 的情况. 很明显, 电导率对电流密度的分布有重大 影响. 对于超导体, $\sigma \rightarrow \infty$, 根据(4)式, 电流只会集中 在两个金属板的外侧表面. 但是如果电流密度超过 该超导体的临界电流密度, $J_{c}$, 则要使超导体失超, 其电导率不再是无限大. 所以对于一对超导体板来 说, $J_{x}$ 分布会如图中蓝色曲线所示.

通过上述分析，可以概括：具有一定频率的交流 电同向通过两个邻近的导体时, 两个金属板内的电 流趋于向其远离另一金属板的方向偏离, 其偏离的 程度与交流电的频率和金属板的电导率有关, 电导 率越高电流的偏离程度也越高. 对于超导体而言, 其 超导态的电导率趋于无限大, 虽然还要受到超导临 界电流的限制, 电流不可能无限向两边集中, 但超导 体中电流分布的偏离程度一定比一般导体大得多, 这就是我们在多层导体的超导电缆中看到的超强邻 近效应的成因。

对于象我们超导样品电缆这样的六层结构, 解 析求解对应的 Maxwell 方程组是我们目前力不能及
的, 所以从数学上证明我们的实验结果的正确性是 困难的. 上面举的一对平行板的例子应给我们一个 很好的启发，定性地支持我们的认识.

我们还可以用下面形象的方法来来进一步解释 我们的结果. 假设有六个相同的长导体平行板相互 间离得很近，那么在它们的长度方向通交流电时，由 于临近效应的作用，电流的分布就会形成由中间板 向两边的板递增的趋势. 如果象图 9 所演示的那样, 将这些平行板弯曲成同轴圆筒，圆筒的轴平行于平
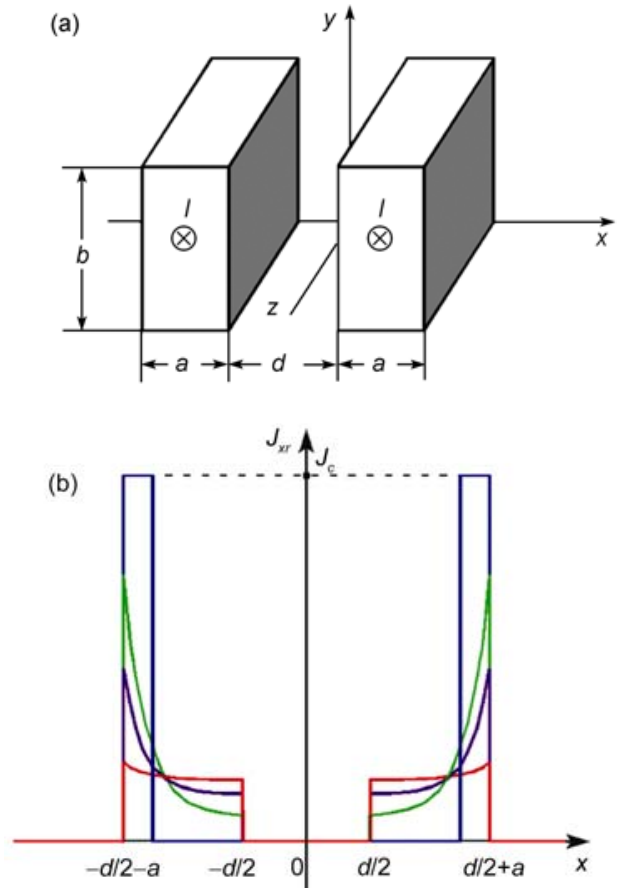

图 8 两个邻近的平行金属板通过交流电流时 所产生的邻近(趋肤)效应示意图

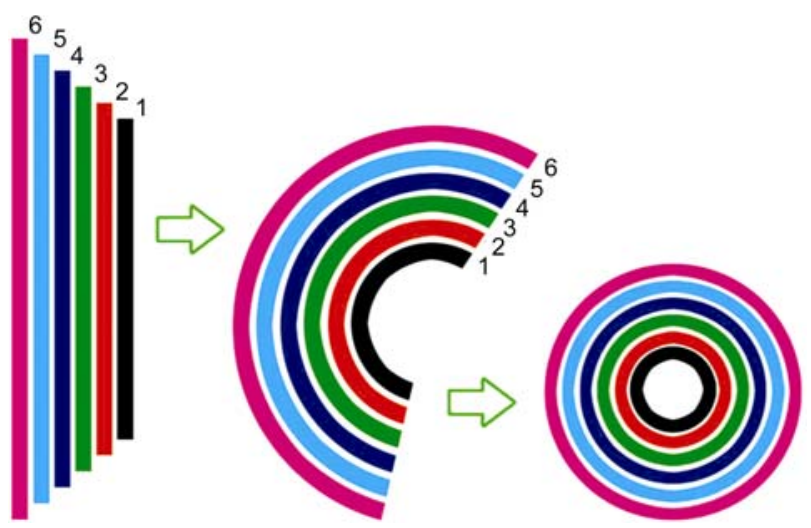

图 9 由六个平行板演绎到六层同心圆示意图 
行板的长度方向. 那么, 在其中最右边的平行板 (1) 就成了同轴圆筒的最内层, 最左边的平行板(6)就成 了同轴圆筒的最外层. 这样一来, 原来这组平行板的 两边就成了同轴圆筒的内、外. 由此来理解为什么在 临近效应很强时, 电流趋向同轴圆筒内、外两侧就变 得比较容易了. 另外, 因为临近效应和趋肤效应是同 一物理作用机制反映在不同几何特征的导体的两个 现象, 所以从内、外对应的两层比较来看, 外部的一 层的电流大于内部的对应层是有道理的. 这也可理 解为圆筒的曲率对洛仑兹力作用效果的影响.

\section{5 结论}

通过对三个超导电缆样品的实验结果的分析, 我们做出以下推论.

1)在两个并联的邻近超导体中, 若通过同向交 流电流，则电流会趋向集中在两导体中的远侧.

2)在多个并联的邻近的超导体中，若通过交流 电流，电流会趋向分布在距离较远的导体中.

3)对于多个邻近的同轴并联超导体薄筒, 如果 筒的直径远大于它们的间距, 那么在通过交流电时, 电流会趋于流在靠近两边 (最外层和最内层为两个边) 的圆筒, 越接近中间的薄筒, 电流会越小. 对于内外
对应的两个薄筒 (从外数第 $K$ 层和从内数第 $K$ 层, $K=$ $1,2, \cdots)$, 外边的电流会更大.

4)对于多层结构的高温超导电缆, 虽然每个导 体层是由分立的带材绕制而成的，但通流时的层电 流分布规律与 3)中描述的类似.

尽管邻近效应形成的原因与导体是否处于超导 态并无关系，但其作用效果却受到导体电导率的强 烈影响. 在我们的实验中, 同样结构的电缆在处于常 导态时, 邻近效应的影响可以忽略不计, 利用常规的 电路计算就可以很好地解释电流的分布规律. 但当 电缆进入超导态后, 电流的分布规律发生了彻底变 化, 无论如何改变绕制参数, 电流比例始终趋向向内 外两侧的导体层递增. 这说明邻近效应的影响已经超 过了其他因素的作用，而主导了超导电缆中的电流分 配, 因此我们把这种在超导体中邻近效应的强烈表现 称为超强的邻近效应. 事实上，一些文献 ${ }^{[20,26,27]}$ 报道 了在超导电缆中相似的层电流分布, 只是没有能够 准确地解释这种现象的成因.

邻近效应会显著影响电流在多个相邻并联超导 导体中的电流分布, 从而影响到超导设备整体的通 流能力、运行损耗等. 在超导电缆和其他涉及到交流 电流的超导设备的设计与制作中, 邻近效应是必须 考虑的重要因素.

\section{参考文献}

1 Terman F E. Radio Engineers' Handbook. Columbus: McGraw-Hill, 1943

2 Dowell P L. Effects of eddy currents in transformer windings. Proceedings IEEE, 1966, 113: 1387-1394

3 Ferkal K, Poloujadoff M, Dorison E. Proximity effect and eddy current losses in insulated cables. IEEE Trans Power Delivery, 1996, 11: $1171-1178$

4 Klontz K W. Skin and proximity effects in multi-layer transformer windings of finite thickness. In: Conference Record of the 1995 IEEE. IAS: IEEE, 1995. 1: $851-858$

5 Howe G O. The high-frequency resistance of multiply-stranded insulated wire. Proc Royal Society London, 1917, 93: 468 - 492

6 Urling A M, Niemela V A, Skutt G R, et al. Characterizing high-frequency effects in transformer windings-—A guide to several significant articles. APEC, 1989, 89: 373-385

7 Evetts J, Cahn R W, Bever M B. Concise Encyclopedia of Magnetic \& Superconducting Materials. Pergamon Press, 1992

8 Petersen T W, Goldfarb R B. Effect of mechanical deformation on Nb-Ti filament proximity-effect coupling at the edges of SSC cables IEEE Trans Magn, 1991, 27: 1809-1810

9 Polak M, Krempasky L, Majoros M, et al. Anomalous magnetization behaviour in fine filamentary NbTi superconducting wires. IEEE Trans Appl Supercon, 1993, 3: 150-153

10 Black R M. The History of Electric Wires and Cables. London: Peter Pergrinus, 1983

11 Terrell C, Wilford S. American Electricans’ Handbook. New York: McGraw Hill, 1987

12 Kawabata S, Terashi D, Tsuji M. Current distributions at steady-state of superconducting cable conductors for ac use. IEEE Trans Appl Supercon, 1999, 9: 587-590 
13 Nikulin A D, Vedernikov G P, Potanina L V. Comparative study of ac superconducting strands and cables with copper-manganese and copper-nickel matrices. IEEE Trans Appl Supercon, 2000, 10: 1231-1234

14 Bolza A, Metra P, Nassi M, et al. Recent developments in HTS power cable applications. IEEE Trans Appl Supercon, 1997, 7: 339-344

15 Sinha U, Hughey R L, Hesterlee J, et al. Design and construction of LN2 cooled prototype superconducting transmission cable. IEEE Trans Appl Supercon, 1997, 7: 351-354

16 Shibata T, Isojima S, Watanabe M, et al. Development of high temperature superconducting power cable prototype system. IEEE Trans Power Delivery, 1999, 14: 182-187

17 Xin Y, Hou B, Bi Y, et al. Introduction of China's first live grid installed HTS power cable system. IEEE Trans Appl Supercon, 2005, 15: $1814-1817$

18 林良真, 肖立业. 高温超导输电电缆的现状与发展. 电力设备, 2007, 8: 1-4

19 Boggs S A, Collings E W, Parish M V. AC losses in HTSC conductor elements. IEEE Trans Appl Supercon, 1992, 2: 117-121

20 Sytnikov V E, Dolgosheev P I, Svalov G G, et al. Influence of the multilayer HTS-cable conductor design on the current distribution. Physica C, 1998, 310: 387-391

21 Leghissa M, Rieger J, Neumuller H W, et al. Development of HTS power transmission cables. IEEE Trans Appl Supercon, 1999, 9: 406411

22 Däumling M. Studies of high temperature superconductors. Narlikar A, ed. Nova Science Publishers, 2000. 73-111

23 李健, 林良真. 高温超导交流输电电缆导体层电流分布研究. 电工电能新技术, 2000, 2: 7-16

24 戴训江, 李敬东, 唐跃进, 等. 高温超导电缆的磁场计算. 华中科技大学学报, 2003, 31(12): 38-40

25 赵臻, 邱捷, 王曙鸿, 宫瑞否. 高温超导交流电缆电流分布及结构优化的研究. 西安交通大学学报, 2004, 38(42): 352 - 356

26 Mukoyama S, Miyoshi K, Tsubouti H, et al. 50-m long HTS conductor for power cable. IEEE Trans Appl Supercon, 1997, 7: 1069-1072

27 Olsen S K, Traholt C, Kuhle A, et al. Loss and inductance in a 4-layer superconducting prototype cable conductor. IEEE Trans Appl Supercon, 1999, 9: 833-836 